\title{
Téoros
}

Revue de recherche en tourisme

\section{L’expérience touristique dans une société nomade} Quel avenir?

\section{Marc Laplante}

Volume 18, numéro 3, automne 1999

Le tourisme dans tous ses états

URI : https://id.erudit.org/iderudit/1071847ar

DOI : https://doi.org/10.7202/1071847ar

Aller au sommaire du numéro

Éditeur(s)

Université du Québec à Montréal

ISSN

0712-8657 (imprimé)

1923-2705 (numérique)

Découvrir la revue

Citer cet article

Laplante, M. (1999). L'expérience touristique dans une société nomade : quel avenir ? Téoros, 18(3), 17-21. https://doi.org/10.7202/1071847ar d'utilisation que vous pouvez consulter en ligne.

https://apropos.erudit.org/fr/usagers/politique-dutilisation/ 


A N A 4 L Y S E

\section{L'EXPÉRIENCE TOURISTIQUE DANS UNE SOCIÉTÉ NOMADE}

QUEL AVENIR ?

\section{Marc Laplante}

* Ainsi, à l'orée des années 90, la littérature est en passe de devenir non seulement voyageuse mais pourvoyeuse - en part de rêve, d'images en temps réel, de réalités virtuelles - des agences de voyages, des multinationales, des télécommunications et autres tour operators de l'esprit. ${ }^{\prime}$

\section{LE NOMADISME D'AGRÉMENT ET LES AUTRES DÉPLACEMENTS}

Il y a vingt ans environ, j'ai cru trouver la signification première du tourisme dans nos cultures postmodernes : le retour à un très vieil etat des terriens, le nomadisme, ce retour se faisant dans le cadre de la vie jouée, celle du temps libre des week-ends et des grandes vacances.

J'ai longtemps suivi cette piste. Un projet de recherche autonome, vers 1981, m'a amené à élaborer une mesure du dépaysement touristique pour construire un test de motivation au voyage. Le nomade, théoriquement, est toujours dépaysé _ ou il ne l'est jamais_s'il définit le monde comme son pays. Le touriste se dépayse (plus ou moins) pour un certain temps; son expérience consiste d'abord et avant tout $a$ partir; puis à vivre autrement, c'est-ă-dire d'abord et avant tout, une fois encore, à se déplacer constamment.

Partir et circuler comme rituels fondateurs de la conduite touristique, au début des années 1980 , n'était déjà plus une idée neuve. Depuis la génération de Jack Kirouac (On the Road) de 1950 à 1960 , depuis les révoltes étudiantes d'Amérique et d'Europe de 1960 à 1970 , depuis l'expansion rapide des organismes de tourisme pour les jeunes de 1970 à 1980 , partir de chez soi, partir pour longtemps, aller loin, géographiquement et culturellement, vivre dans ses bagages et être toujours itinérants n'étaient plus des gestes d'exception chez les jeunes générations. Et la culture-jeune influençait toute la culture. A partir des années 1960 dans les societés industrialisées (un peu plus tard au Québec), les déplacements se développent : voyages d'affaires ou d'études, tourisme sous des formes de plus en plus variées, déplacement des vedettes, des artistes, des intellectuels, etc. Le voyage s'installe définitivement comme un élément de style de vie pour plusieurs catégories de population.

Parallèlement, certains déplacements s'intensifient et deviennent des faits de civilisation de notre temps : retour en France des Français d'Algérie, arrivées des boat people et d'autres réfugiés sur tous les continents, migrations massives vers les grandes villes, masses humaines jetées sur les routes par les guerres, entrées clandestines de milliers de Latino-Américains aux États-Unis et au Canada, etc. Le bilan d'un tel phénomène reste difficile à faire, mais l'idée que toute la population de la planète est en déplacement s'installe !

Sans y voir de relations de cause à effet, on peut souligner des corrélations : les voyages d'agrément deviennent un phé- nomène de masse en même temps que s'accroissent les déplacements de personnes dans le monde entier, déplacements obligés et plutôt désagréables, trop souvent.

Faut-il alors simplement constater, comme il y a vingt ans, que le nomadisme reprend ? On vient de souligner que toutes les sociétés semblent de moins en moins sédentaires. En matière de tourisme, il n'y a pas suffisamment d'études spécialisées pour conclure que le nomadisme est devenu le mode de vie principal des amateurs du voyage d'agrément. Comment explorer, indirectement, les changements survenus dans l'univers, plutôt secret, des aspirations et des valeurs? Je vais tenter l'exploration en suivant le sort d'un concept clé des écrits sur le tourisme depuis deux décennies, celui d'expérience touristique. Depuis le temps que je ferraille avec cette notion, je témoignerai qu'on peut s'en servir pour sonder l'avenir, comme fait le sourcier avec une baguette de sureau pour trouver de l'eau ! On suivra quelques pistes pour faire de la prospective en attendant l'ouvrage qui apportera une nouvelle synthèse du nomadisme d'aujourd'hui dans notre monde et dans nos systèmes de valeurs ${ }^{2}$.

\section{L'EXPÉRIENCE TOURISTIQUE : UNE OCCASION DE JOUER AU NOMADE}

Pour les fins d'une enquête sur les pratiques, les besoins et les aspirations touristiques des Québécois, entreprise avec le géographe Roger Nadeau de l'Université de Sherbrooke en 1980 , il a fallu définir 
d'une façon opératoire (i.e. pour des études par questionnaire et entrevue) la notion d'expérience touristique. A ce momentlà, on trouvait l'expression dans quelques écrits américains seulement, par exemple, dans des articles d'Éric Cohen, de Jafar Jafari et de quelques autres ${ }^{3}$; un essai dominait les études, celui de Dean MacCannell, publié en $1976^{4}$.

Le concept d'expérience, selon MacCannell, est utile pour designer de façon globale ce qui est vécu par une personne en situation de voyage d'agrément. A l'instar de cet auteur, nous avions déjà choisi de réserver le nom de touristes aux seules personnes qui consacraient l'essentiel de leur voyage d'agrément au nomadisme. L'expression expérience touristique servait donc à désigner la totalité de ce vécu de nomadisme de vacances. MacCannell proposait de lire cette totalité comme une production cul. turelle : culturelle parce que faite essentiellement de produits symboliques (non matériels, intangibles, comme le feeling, l'émotion, le plaisir, l'exotisme, etc.); production parce que constituée de nombreux produits particuliers, assemblés d'une façon particulière, montés comme on monte un film avec des bouts de pellicules. Ainsi abordées, les expériences touristiques étaient des séquences du temps du déplacement ; elles pouvaient durer une heure, trois heures, une journée, plusieurs jours. Plus on les a étudiées, plus on a vu comment elles s'emboîtaient les unes dans les autres comme des poupées russes ; finalement, il devenait évident que la notion d'expérience touristique recouvrait toutes les expériences vécues en cours de route.

On valorisait ainsi la totalité du voyage d'agrément en l'abordant comme une expérience de nomadisme ${ }^{5}$; $c^{\text {'est en tant }}$ que nomade en train de traverser une société et une culture étrangère à la sienne que le touriste atteint le monde du nonordinaire, pour employer un beau concept de J. Jafari ${ }^{6}$, et y reste un certain temps. Ainsi, au terme de cet exercice de conceptualisation. I'expérience touristique est-elle devenue cette expérience qui consiste à partir volontairement de chez soi pendant un certain temps pour aller vers le monde du non-ordinaire et vivre ainsi, dans cet espace-temps non-ordinaire (espace inconnu ou moins connu, temps exceptionnel de congé prolongé et d'obligations réduites, etc.), tous les événements

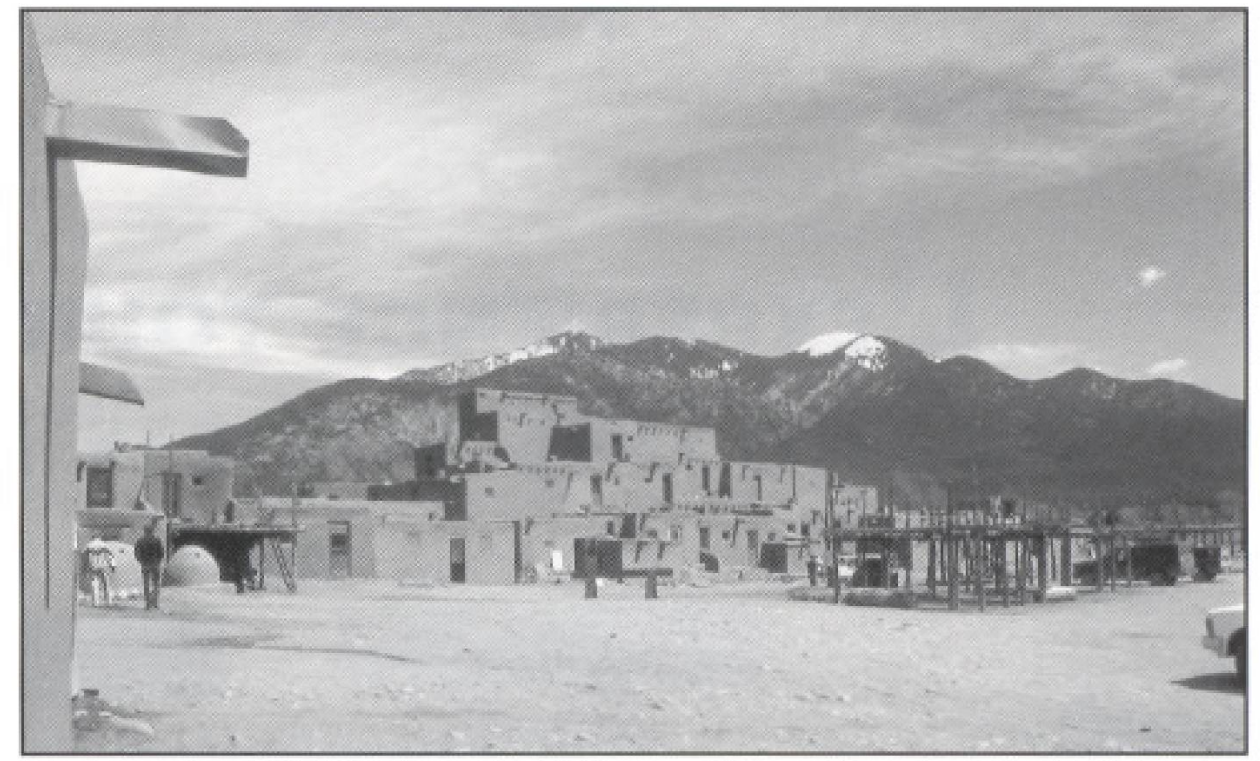

La rencontre de "l'indigène à Taos Pueblo (Nouveau-Mexique) : la * valeur ajontée w de la station de ski qui domine le pillage.. (photo Lue Noppen)

qui peuvent survenir au cours de la traversée des espaces du nomadisme de plaisance ou d'agrément.

Prolongeant les vues des collègues americains, je leur ai ăussi attaché les résultats d'une quinzaine d'années de recherches en sociologie du temps libre. J'ai ajouté à la conception de l'expérience touristique présentée ci-dessus quelques concepts clés de la théorie des jeux de Roger Caillois ${ }^{7}$ qui m'inspiraient depuis longtemps : dans l'espace-temps spécifique et unique du voyage d'agrément, tous les comportements des touristes sont des comportement ludiques. J'ai consacré beaucoup de moyen de recherches à l'observation directe, in sim, des comportements des touristes et je n'ai plus aujourd'hui de réserves pour conclure que l'expérience touristique appartient totalement à l'univers de la fête et du jeu et, pour l'essentiel, le jeu consiste à jouer au nomade. L'expérience ludique a son espacetemps propre, comme nous l'avons déjà vu, et satisfait également à tous les autres critères de la théorie de Caillois ${ }^{8}$.

\section{L'EXPÉRIENCE TOURISTIQUE RÉCUPÉRÉE...}

Il n'y a pas lieu de raconter ici comment les conceptions du tourisme comme phénomène socioculturel ont généré, notamment, de nouvelles recherches, des enseignements spécialisés, des conférences, des colloques, ni comment, graduellement, une telle interprétation a servi la promotion du tourisme culturel. Pour avoir démontré et répété constamment que toute expérience touristique est une expérience culturelle, je ne devrais pas m'étonner maintenant du success du concept de tourisme culturel. J'aurais même dû prévoir que les responsables de l'offre touristique, dans le cadre d'une diversification de leurs produits qui s'imposait, allaient bientôt offrir la culture, avec ou sans la nature!

Dans la pratique, la gamme des activités offertes aux touristes s'est élargie; le monde des arts, du spectacle, des expositions et des musées a pu trouver dans le porte-monnaie des touristes des revenus que l'Etat n'assurait plus et, dans certains cas, ces opérations touristiques ont pu sauver des lieux et des biens patrimoniaux. On pourrait cependant s'inquiéter du traitement de plus en plus mercantile de la culture, surtout dans le cadre des mondialisations dont plusieurs touchent le champ culturel, mais je sortirais trop de mon propos en approfondissant ces questions.

Je m'inquiéterai plutôt du sort réservé récemment au concept d'expérience touristique qui sert maintenant à désigner des produits, à decrire une offre touristique regroupant des produits et des activités ! Les touristes vivent des expériences touristiques, mais peut-on imaginer des forfaitistes (packagers) organisant toute une expérience touristique pour leurs clients ? On sait qu'ils peuvent organiser. pour un prix fixe, un voyage qui comprend le transport, l'hébergement, la restauration. un tour de ville, une excursion et quelques 
autres prestations ; on sait qu'ils fabriquent des voyages sur mesure, autour d'un thème (les vignobles, par exemple) ; on connaît leur circuit-spectacle, etc. Mais l'expérience touristique, comprise comme une totalité, marquée par l'esprit ludique, née précisément du nomadisme, n'est-elle pas une réalisation trop complexe et trop intangible pour que l'on puisse l'organiser, la \& préassembler $\%$, evaluer ses coûts, etc.?

Cela risquerait de réduire le sens et la richesse de cette notion, comme en fait foi la citation suivante fondée sur les conclusions d'un Forum sur l'industrie touristique du Québec, tenu en mars 1997

Le passage de la notion de produit louristique à un concept d'expérience touristique intégrant les produits et les activités répond aux changements des clienteles et au degré de maurité atteint par l'industrie touristique québécoise."

En conséquence, offrir désormais des expériences touristiques plutồt que des produits fait partie du « premier axe d'intervention de l'orientation stratégique deux $\$$ de cette politique, ou vice versa ! Ce qui est certain pour les auteurs, c'est que ce changement est une réponse à des changements de clientèles que ce document si stratégique, devenu aujourd'hui une bible pour toutes les interventions touristiques au Québec, ne précise pas beaucoup cependant. Pour dire quoi que ce soit sur l'experrience touristique, son passé, sa situation présente ou son avenir, il faut done nécessairement revenir au client, à celui ou à celle qui consomme des expériences touristiques.

\section{L'EXPÉRIENCE : UNE PRIME À LA CONSOMMATION}

MacCannell, qui a introduit la notion d'expérience touristique, a longuement analysé les sociétés et les cultures américaines et européennes postmodernes avant d'examiner le rôle et le sens du tourisme dans de tels ensembles socioculturels. Résumons sommairement sa pensée sur ce point essentiel : du point de vue d'un industriel et d'un commerçant, offrir' des expériences est une stratégie publicitaire pour faire vendre des biens et des servi- ces ; l'expérience accompagne le produit à vendre comme une sorte d'aura, de halo, pour le rendre encore plus séduisant. Par exemple, on n'offre plus une automobile, mais une sensation de liberté (ou de puis= sance, ou de...) au volant de tel engin. Sensation, feeling, émotion, extase, vertige, etc., voilà des expériences que ne permet plus le quotidien, mais qu' on peut obtenir avec l'achat de tel bien ou service.

Cette stratégie de marketing fonctionnait très bien dans les années 1970 avec à peu près tous les biens et services, mais elle s'était révélée particulièrement efficace pour la vente des produits touristiques. On peut comprendre facilement pourquoi si I'on se rappelle que les produits touristiques sont rarement tangibles; ils appartiennent aux produits symboliques, immatếriels : le plaisir de voir un bon film, la satisfaction d'un bon repas, l'excitation d'un party, la sensation du paradis sous les palmiers dans les mers du sud, etc. Qu'estce que le consommateur achète quand il paie pour de tels produits? Il achète avant tout une expérience, un feeling, un plaisir. La place prédominante du symbolisme dans les sociétés les plus développées d'aujourd'hui est le phénomène central qui devrait retenir l'attention. Faut-il y voir le contrepoids de la prépondérance du matérialisme dans les cultures dites postmodemes ? Peut-être; la question reste ouverte !

Décider aujourd'hui d'appeler experience ce que l'industrie touristique offre à ses clients relève d'abord du soin de son image : en présentant ainsi ses biens et services, elle veut peut-être donner l'impression d'offrir des produits de qualité ! Je crois que l'industrie touristique, actuellement, cherche à faire feu de tout bois. Serait-elle inquiète pour l'avenir ? Percevrait-elle déjà que le nomadisme d'agrément est une activité qui déborde de plus en plus le temps des week-ends et des grandes vacances? Avant de revenir à ces interrogations, j'aimerais bien d'abord qu'on se rappelle, une fois de plus, de l'origine du tourisme.

En remontant dans l'histoire socioculturelle du tourisme, on trouve que l'offre touristique a toujours été l'offre d'une expérience, au sens phénoménologique du terme; on peut s'en persuader rapidement en feuilletant de vieux guides touristiques. Corbin ${ }^{10}$ raconte, par exemple, comment on conseillait la pratique de la peinture sur le rivage ; les livres-guides disaient comment il fallait peindre la mer, où il fallait se placer pour en saisir toute la puissance, à quelle heure du jour, par quel temps et quel vent il fallait la capter, etc. Des guides contenant de telles descriptions existaient au milieu du XVIII ${ }^{\circ}$ siècle, à la naissance du tourisme ou presque. Urbain ${ }^{11}$ a multiplié les descriptions d'expériences à vivre par les touristes, non seulement sur la plage, mais dans tous les espaces types qui les attirent.

Le plus important, ce n'est pas l'intégration des produits et des activités, comme le mentionne explicitement le texte sur la politique touristique québécoise, cité ci-

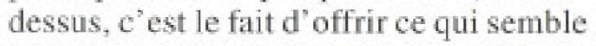
être des packages d'émotions, de sentiments, de plaisir. L'auteur du guide montre comment s'y prendre pour ressentir telle ou telle chose, pour expérimenter telle émotion... Il ficelle, il encode, en fait, des façons de sentir, de percevoir et, finalement, d'apprecier, de jouir. Avec le temps, les promoteurs touristiques ont emmagasiné ainsi une multitude d'interventions qui ont plu à des touristes et qu'on trouve toujours aujourd'hui dans les livres-guides, dans les discours et les gestes des guides-accompagnateurs et des animateurs, etc. Avec le concept d'expérience touristique, on a l'impression que les intervenants ont enfin trouvé un mot passepartout pour présenter leurs produits.

\section{MAIS NOTRE CONCEPT-VEDETTE N'A PAS DIT SON DERNIER MOT...}

Une lecture attentive de bon nombre de vieux livres-guides touristiques permet de découvrir que les expériences ainsi préparés à l'intention des touristes étaient d'autant plus attirantes et irrésistibles qu'elles prenaient quelque saveur de fruit défendu. Le temps du voyage d agrément, situé hors du temps, l'espace du voyage d'agrement s'apparentant à un non-espace (le terrioire du vide, dira Corbin à propos du rivage), les expériences possibles dans cet espace-temps non-ordinaire pouvaient, elles aussi, sortir de l'ordinaire, comme on l'a déjà dit. Les aspects piquants de ces expériences tenaient beaucoup au fait de vivre hors normes pour un certain temps. L' éloignement physique du milieu social habituel, les déplacements fréquents, le caractère excentrique des lieux de séjour de vacances, les rituels qu'il fallait 
apprendre pour fréquenter ces lieux, tout cela contribuait à rendre attirante l'expérience touristique.

Pour comprendre qu'on se soit excité pour si peu, selon nos règles sociales actuelles, rappelons-nous que ces debauches touristiques se sont développées alors que le puritanisme se maintenait au sommet de son pouvoir dans la société anglaise des années 1750-1800. J'ai toujours eu des incrédules parmi mes étudiants quand je démontrais que le droit au plaisir était une conquête du $X^{\circ}$ siècle... Donc, pendant longtemps, l'expérience touristique a gardé un goût d'aventures dans des jardins aux fruits plus ou moins défendus ! Jusqu'à la lecture de l'énoncé de politique touristique pour le Québec, je cherchais naîvement, moi aussi, de telles saveurs dans mes experiences de voyages de vacances...

Je viens de définir ainsi, par étape, ce que j'ai pu appeler, dans un ouvrage de synthèse $^{12}$ l'expérience touristique originale. Cependant, les récents glissements de sens de cette notion l'ont affadie et banalisée, ce qui permet de dire maintenant qu'on offre une expérience touristique parce qu'on assemble un forfait comprenant deux repas, une nuitée, un transport en car, une entrée dans un musée et un plan pour faire un tour du vieux quartier à pied!

Pour rester dans l'esprit original du concept d'expérience touristique, il faudrait aujourd'hui réserver cette expression aux voyages d'agrément entraînant, pour les touristes, de grands dépaysements physiques, psychologiques, sociaux, religieux, politiques... Il faudrait aussi que ces voyages redeviennent de longues routes transversales amenant finalement les touristes à sortir de leurs habitudes acquises de penser, de vivre, d'aimer, de voir et de comprendre. Mais, selon les entendements courants, le mot touriste $\mathrm{s}^{*}$ appliquerait-t-il encore à ces voyageurs, plus proches du pèlerin, de l'itinérant ou du grand nomade que du vacancier ? Si oui, c'est alors le fournisseur de prestations touristiques qui devra abandonner son nouveau concept promotionnel.

\section{FUTURS NOMADES ET NOMADES DU FUTUR}

Ces réflexions sur l'évolution des usages du concept d'expérience touristique rappellent qu'on aura toujours à trouver des mots pour dire les choses nouvelles. Mais aujourd'hui, à propos du tourisme, $1^{+}$enjeu est moins au niveau du langage, peutêtre, qu'à celui de ses futurs rapports avec la société.

Imaginons la tendance lourde suivante qui se confirme et se maintient au cours des 20-25 prochaines années : pour étudier, pour travailler, pour se reposer, pour se divertir, tous ceux et celles qui peuvent bouger dans notre société bougent, vont et viennent par-delà les frontières géopolitiques et socioculturelles, vivent constamment branchés sur le monde entier grâce aux nouvelles technologies de l'information et des télécommunications, etc. Le sédentaire volontaire est le cas d'exception. Dans ce contexte, partir de chez soi pour consacrer quelques semaines au nomadisme va-t-il constituer toujours une expérience touristique, au sens que j'ai donné à cette notion? Ne faudra-t-il pas que cette expérience, pour garder, par exemple, son caractère exceptionnel, propose du tourisme extrême? On a déjà des sports extrênes, des party extrêmes; le tourisme sexuel n'est-il pas dejà une forme de tourisme extrême?

J'ai commencé à chercher dans cette voie après avoir approfondi les tribus de J.D. Urbain et avoir tentế d'associer chaque tribu à des façons différentes de fréquenter les grands espaces symboliques de la Ville, de la Campagne et du Désert ${ }^{13}$. L'auteur a trouvé dans les promotions et les publicités touristiques des années 1980 des appels à des clientèles nouvelles pour le tourisme, celles du tourisme expérimental.

Dans son modèle, Urbain situe les expériences touristiques sur divers continuums dont l'un va du rite au risque : visiter le Vieux-Montréal en car appartient au tourisme ritualise ; descendre les Rapides-deLachine en canot pneumatique comporte déjà davantage de risques; partir pour l'Ungava en hiver, derrière un traîneau tiré par des chiens, exige un bon gout du risque. Les tribus du tourisme expérimental ont plutôt le goût du risque.

Urbain a identifié deux tribus de ce genre: les avertis et les ascèles; la première regroupe les amateurs $d^{\prime}$ un tourisme aux limites du monde possible et la seconde, ceux et celles qui veulent aller au bout d'eux-mêmes. Schématiquement, les avertis vont préférer les villes disparues, les villes impossibles comme Brasilia, ou encore les villes dangereuses (guerre, drogue, etc.) ; leur campagne est pleine de vestiges, de fouilles archéologiques révélant des présences anciennes ; leur désert est vu comme l'espace du divin ou la pure nature, celle d'un monde inhumain ou préhumain, lieu également de la tentation du vide. Les ascètes aiment aussi aller loin, psychologiquement ou autrement. Ils vont aller à la ville pour y courir un marathon et à la campagne pour la traverser en cyclotourisme; les lieux qui symbolisent le désert les attirent et ils y font une sorte de tourisme de purification : exotisme de l'aride, signe de mort, attente d'illumination, arrachement au social... ${ }^{14}$

Toutes ces intuitions sur le tourisme dit expérimental reposent sur des indices que l'auteur a pu rassembler vers 1990 ou avant. Ces tribus ne sont certainement plus aussi rares et marginales aujourd' hui qu'il y a une dizaine d'années.

En suivant une telle ligne de prospective, est-ce qu'on peut interroger l'avenir de l'industrie touristique? II y aura, c'est presque certain, de plus en plus de gens à transporter, à loger et à nourrir. A ce propos, l'idée que les nouvelles technologies de communication vont réduire les déplacements physiques est aussi primaire que celle annonçant la réduction du papier a cause des ordinateurs ! Y aura-t-il autant ou plus encore de voyages de vacances organisés, ritualisés ? C'est, à mon avis, ce qui risque le plus de changer à la baisse : les expérimentaux de J.D. Urbain vont augmenter fortement sans se laisser organiser par des opérateurs touristiques, car ces touristes aussi sont expérimentés; ils ont dejà beaucoup voyagé et, s'ils continuent à le faire, c'est pour y trouver, encore et toujours, du non-ordinaire. On n'en sort pas!

Alors, pour faire des affaires, j'irais investir, personnellement, dans des lieux qui ne servaient jadis qu'aux pèlerins ou aux grands voyageurs devant l'Éternel : couvents, monastères, presbytères, églises, grottes, etc., situés aujourd'hui hors circuits. L'aventure touristique du futur, en somme, investira ces lieux où bien des hommes et des femmes avant nous ont compris que les voies du Seigneur étaient impénétrables!

Retiré de l'enseignement depuis twois ans, Marc Laplante est professeur associé au Desparte- 
ment d'études urbaines et touristiques de l' Université du Québec à Montréal et conseiller en recherche.

\section{NOTES}

I Hutchison, Patrick (1992), Nomades, Paris, Noël Blanditi, p. 11.

2 Actuellement, on trouve de multiples sens au mot nomade dans les publications. L'ouyrage d'Hutchison est une réédition d'un numéro de la revue Détours d'écriture, parue en 1983. On y trouve 300 pages de poèmes, d'essais, de récits, d'entrevues, etc., qui sont fideles au titre de la publication. Mais. sous le méme mot-clé. j'ai trouvé un bel ouvrage illustré paru en 1997 chez Buchet Chastel à Paris, intitule Le grand duel : esprit nonade, culture sédentaire, de Pierre De Lagarde, comprenant plus de 400 pages consactées à la dualité nomade-sédentaire dans les domaines de l'histoire, des arts, des sciences, de la religion et même de la politique. Le sédentaire favorise l'ordre, donc le classicisme, alors que le nomade privilégie le mouvement, done l'innovation ; Rome, Ra- phaël, Mozart, Racine pour les sédentaires, contre les Vikings, Michel-Ange, Beethoven, Shakespeare pour les nomades...

3 On consultera les textes suivants pour voir l'évolution des concepts sur 5-10 ans : Cohen, Éric (1985), \& The Sociology of Tourism : Approaches, Problems and Findings: Anmual Review of Sociology, $n^{\circ} 10, \mathrm{p}, 373-$ 392 ; Jafari, Jafar (1985), The Tourist System. A Theoritical Approach to the Study of Tourism. Ann Arbor, Michigan University Microfilm International.

4 MacCannell, Dean (1976), The Tourist. A New Theory of the Leisure Class, New-York, Shocken Books.

5 Contrairement à l'habitude, toujours persistante, de découper ce voyage d'agrément en tranches de saucisson pour n'étudier (un grand mot!) que les sorties, les visites, la pratique d'un sport, etc. Des pièces détachées qui ne donnent pas une idée de l'ensemble auquel elles apparticnnent.

6 Voir le rêsumé de sa thèse dans Loisir et sociéte, Sainte-Foy, Presses de l'Université du Québec, vol. 11, $\mathrm{n}^{\mathrm{1}} 1,1988$, figure 2, p. 62.
7 Caillois, Roger (1955), Les jeur et les hommes, sc edition, Paris, Gallimard.

8 Voir à ce sujet Laplante, Marc (1997), Lexpérience touristique. Fondements sociaux ef culturels, Sainte-Foy, Presses de l'Université du Québec, p. 19 et suivantes : a le jeu source de l'agrément du voyage touristique $\%$.

9 Tourisme Québec (1997), Consultation pour une politique du développement touristique du Québec, p. 12

10 Corbin, Alain (1988), Le territoire du vide. L'Occidente é le désir du rivage, Paris, Flammarion/Aubier (coll. Champs).

11 Urbain, Jean-Didier (1994), Sur la plage. Maurs et coutumes baltiéaires, Paris, Plon et Rivages. Voir aussi, paru en 1991, L'idiot du voyzge. Histoires de touristes, Paris, Plon.

12 Laplante, Mare (1997), op. cit., chapitre 3, p. $77-100$.

13 Laplante, Marc (1994), Les trois espaces de la carte du touriste, selon J.D. Urbain ; contribution a l'étude touristique des relations entre centres et périphéries $\%$, Téoros, vol. $13, \mathrm{n}^{\mathrm{5}} 1, \mathrm{p}, 40-45$.

14 ldem, tableau, p.44.

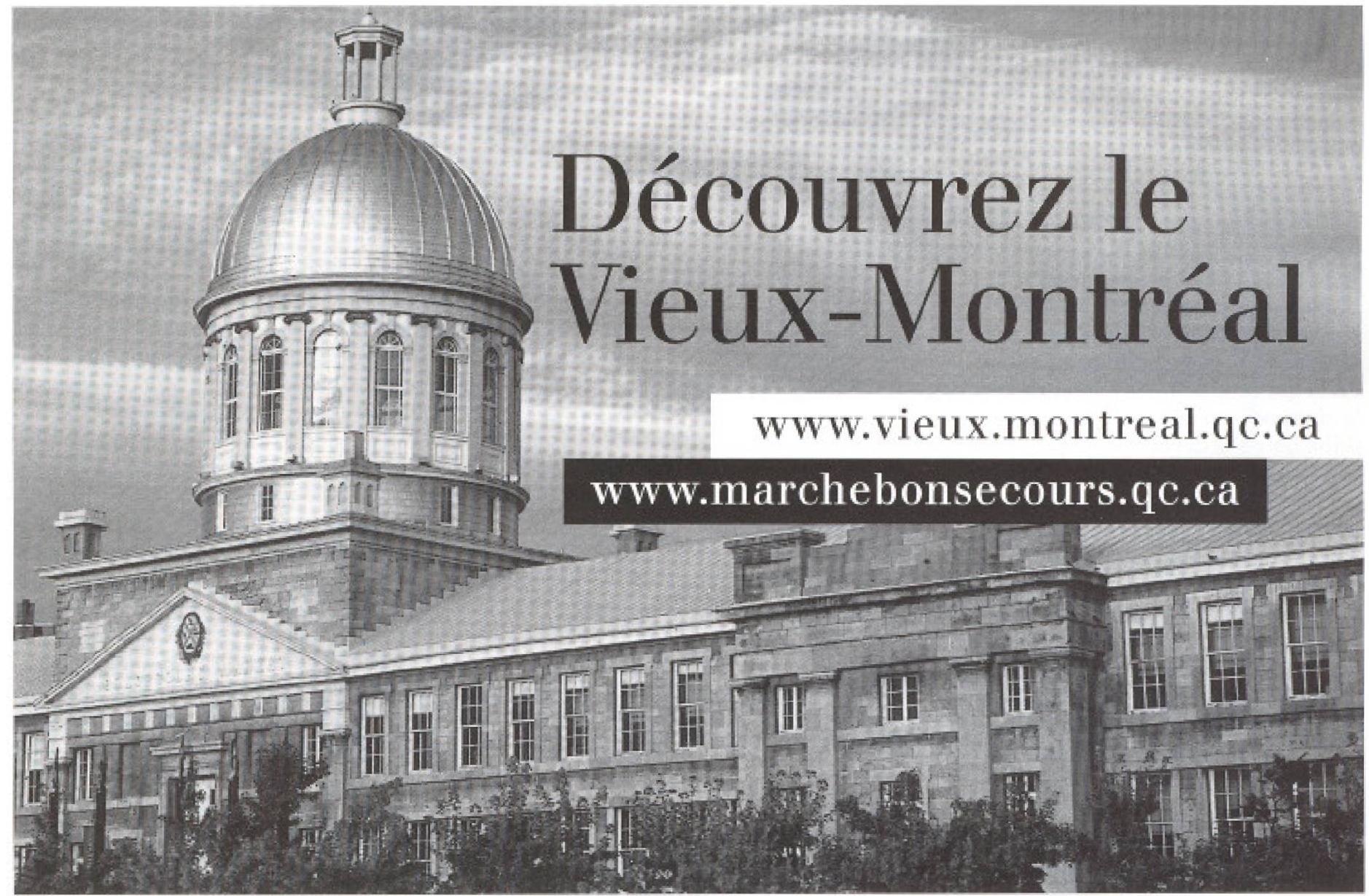

